\title{
THE HELSON-SARASON-SZEGO THEOREM AND THE ABEL SUMMABILITY OF THE SERIES FOR THE PREDICTOR
}

\author{
MOHSEN POURAHMADI ${ }^{1}$
}

\begin{abstract}
It is shown that the best linear least squares predictor of a stationary stochastic process has a mean Abel summable series representation in the time domain if its density satisfies the condition of the Helson-SarasonSzego theorem. This provides an answer to an open question of Wiener and Masani (1958) in prediction theory.
\end{abstract}

1. Introduction. Let $H$ denote the Hilbert space of complex-valued random variables with zero expectations and finite variances. The inner product in $H$ is defined by $(X, Y)=E X \bar{Y}, X, Y \in H$.

An important problem in the theory of linear least squares prediction of a purely nondeterministic weakly stationary stochastic process (WSSP) $\left\{X_{n}\right\}_{n=-\infty}^{+\infty}$ in $H$ is to find conditions on its spectral density $w$ such that the best linear least squares predictor of $X_{\nu}(\nu \geq 1)$, denoted by $\hat{X}_{\nu}$, based on the past and present observations, i.e. $X_{k}, k \leq 0$, can be written as $\hat{X}_{\nu}=\sum_{k=0}^{\infty} a_{k} X_{-k}=\lim _{N \rightarrow \infty} \sum_{k=0}^{N} a_{k} X_{-k}$, where $a_{k}=\sum_{j=0}^{k} c_{\nu+j} d_{k-j}$ with $c_{k}$ and $d_{k}$ being the $k$ th Fourier (Taylor) coefficients of $\phi$ and $\phi^{-1}$, respectively, with $\phi$ being the optimal factor of $w, \mathrm{cf}$. $[\mathbf{1}, \mathbf{8}]$. If such a representation for $\hat{X}_{\nu}$ exists, then we say that the best linear least squares predictor of the process has a mean-convergent autoregressive series representation in the time domain $[8]$.

It was first shown by Wiener and Masani $[8]$ in 1958 that if

$$
0<c \leq w \leq d<\infty, \quad \text { a.e. (Leb.), }
$$

then $\hat{X}_{\nu}$ has a mean-convergent autoregressive series representation. Later, Masani [5] weakened the severe boundedness condition in (1) and replaced it by

$$
w \in L^{\infty} \text { and } w^{-1} \in L^{1} .
$$

For $1 \leq p \leq \infty, L^{p}\left(H^{p}\right)$ denotes the usual Lebesgue (Hardy) space of functions on the unit circle in the complex plane and for a density function $w, L^{2}(w)$ stands for the Hilbert space of measurable functions $f$ on the unit circle with $\int_{-\pi}^{\pi}|f|^{2} w d \theta<$ $\infty$.

Masani's condition $w \in L^{\infty}$ is also restrictive. By using a deep result of Helson and Szego $[\mathbf{2}]$ the author has shown $[\mathbf{6}]$ that if

$$
w=e^{u+\tilde{v}},
$$

Received by the editors December 10, 1982 and, in revised form, September 27, 1983.

1980 Mathematics Subject Classification. Primary 60G25; Secondary 42A24.

${ }^{1}$ This research was supported in part by the NSF Grant MCS-8301240

(C) 1984 American Mathematical Society $0002-9939 / 84 \$ 1.00+\$ .25$ per page 
where $u$ and $v$ are bounded real-valued functions with $\|v\|_{\infty}<\pi / 2$, then $\hat{X}_{\nu}$ has a mean-convergent autoregressive series representation in the time domain. It is easy to check that densities $w$ exist which satisfy (3) but for which $w \notin L^{\infty}$, cf. [6]. This shows that the boundedness condition in (2) can be relaxed.

Both conditions (2) and (3) require that $w^{-1} \in L^{1}$. Motivated by a yet open question of Wiener and Masani [8, p. 123], concerning the summability of the best linear least squares predictor of a purely nondeterministic WSSP, in the following theorem we show that the condition $w^{-1} \in L^{1}$ can be relaxed and replaced by a weaker condition, if one replaces the requirement of mean-convergence of the linear predictor by the mean Abel summability of the series involved.

2. THEOREM. Let $\left\{X_{n}\right\}_{n=-\infty}^{+\infty}$ be a purely nondeterministic WSSP with the spectral density function $w$ and $\hat{X}_{\nu}$ the best linear least squares predictor of $X_{\nu}(\nu \geq$ 1) based on $X_{k}, k \leq 0$. Then, $\hat{X}_{\nu}=\lim _{r \rightarrow 1} \sum_{k=0}^{\infty} r^{k} a_{k} X_{-k}$, if

$$
w=|P|^{2} e^{u+\tilde{v}},
$$

where $u$ and $v$ are as in (3) and $P$ is an analytic polynomial of some degree $n$ such that all of its roots lie on the unit circle in the complex plane.

3. Remarks. (a) Densities satisfying (3) and (4) were first studied by Helson and Szego $[\mathbf{2}]$ and Helson and Sarason $[\mathbf{3}]$ in connection with the following important problem. Let $H(X, 0)=\overline{\mathrm{sp}}\left\{X_{k} ; k \leq 0\right\}$ and $H^{n}(X)=\overline{\mathrm{sp}}\left\{X_{k} ; k \geq n\right\}, n=1,2, \ldots$ As a measure of the cosine of the angle between the two subspaces $H(X, 0)$ and $H^{n}(x)$ in $H$ define $\rho_{n}=\sup |(X, Y)|$, where the supremum is taken over all $X, Y$, as elements of the unit balls of $H(X, 0)$ and $H^{n}(X)$, respectively. It is easy to see that $0 \leq \rho_{n} \leq 1$. For fixed $n \geq 1$, it is said that $H(X, 0)$ and $H^{n}(X)$ are at positive angle if $p_{n}<1$. The problem of characterizing WSSP's or densities $w$ such that $\rho_{n}<1$ is solved in [2] for $n=1$ and in [3] for $n>1$. The results of these authors, known as the Helson-Sarason-Szego theorem, state that $\rho_{n}<1$, if and only if $w=|P|^{2} e^{u+\tilde{v}}$, where $P$ is an analytic polynomial of degree $n-1$ and $u, v$ are as before. It is easy to check that a $w$ satisfying (4) does not necessarily have the property $w^{-1} \in L^{1}$.

(b) Densities $w$ satisfying (4) have the property that $w \in L^{1}$ and $|P|^{2} / w \in L^{1}$. It is of interest to know whether the converse is true. A positive answer will provide a condition weaker than (4) for the Abel summability of the linear predictor. Also it is of interest to know whether the mere assumption of pure nondeterminism, that is $w \in L^{1}$ and $\log w \in L^{1}$, will imply the Abel summability of the linear predictor.

Proof of Theorem 2 follows from the next three lemmas.

4. LEMMA. Let $\left\{X_{n}\right\}, w$ and $\hat{X}_{\nu}$ be as in Theorem 2 , $\phi$ the optimal factor of $w, \phi_{\nu}$ the spectral isomorph of $\hat{X}_{\nu}, H^{2}(w)=\overline{\operatorname{sp}}\left\{e^{i n \theta} ; n \geq 0\right\}$ in $L^{2}(w), P_{r}(\theta)=$ $\left(1-r^{2}\right) /\left(1-2 r \cos \theta+r^{2}\right)$, and $\phi_{r}(\theta)=\left(P_{r} * \phi\right)(\theta), 0 \leq r<1$. Assume that

$$
\int_{-\pi}^{\pi} P_{r}(\theta-x)\left|\frac{\phi(x)}{\phi_{r}(x)}\right|^{2} d x \leq C
$$

for all $0 \leq r<1$ and $-\pi \leq \theta \leq \pi$, where $C$ is constant. Then:

(a) $\phi_{\nu}$ is of Nevanlina class and so has Fourier (Taylor) coefficients $a_{k}, k \geq 0$ (for the definition of $a_{k}$ 's see $\S 1$ ).

(b) The series for the linear predictor of $X_{\nu}$ is mean Abel summable, if and only if $\lim _{r \rightarrow 1}\left\|\sum_{k=0}^{\infty} r^{k} a_{k} e^{i k \theta}-\phi_{\nu}\right\|_{L^{2}(w)}=0$. 
PROOF. Since $\phi_{\nu}$ is the spectral isomorph of $\hat{X}_{\nu}$, it belongs to $H^{2}(w)$ and $\phi_{\nu} \phi \in H^{2}$. Hence $\phi_{\nu}$ is of Nevanlina class [9, p. 271] and (a) follows. A similar argument shows that all functions in $H^{2}(w)$ are of Nevanlina class, this fact is needed in Lemma 5. (b) is an immediate consequence of the isomorphism between the time and spectral domains of WSSP; cf. [1, p. 563].

The following lemma is a slightly different version of Theorem 1 of Rosenblum [7].

5. LEMMA. The Fourier series of all functions in $H^{2}(w)$ are Abel summable in the norm of $L^{2}(w)$, if and only if $\phi$ ( $\phi$ is the optimal factor of $w$ ) satisfies (5).

6. LEMMA. (a) If $w_{0}=e^{u+\tilde{v}}$, with $u$ and $v$ as in (3), then $w_{0}$ satisfies (5).

(b) If $w_{0}$ is as in (a) and $w=|Q|^{2} w_{0}$, where $Q$ is a polynomial of some degree $n$, then $w$ satisfies (5).

PROOF. (a) It follows from [2, p. 131] that if $w_{0}=e^{u+\tilde{v}}$, then the Fourier series of all functions in $H^{2}\left(w_{0}\right)$ are convergent in the norm of $L^{2}\left(w_{0}\right)$. Thus, from the regularity of the Abel summability method, it follows that the Fourier series of all functions in $H^{2}\left(w_{0}\right)$ are Abel summable in the norm of $L^{2}\left(w_{0}\right)$. That $w_{0}=$ $|\phi|^{2}$ ( $\phi$ being the optimal factor of $w_{0}$ ) satisfies (5) is a consequence of Lemma 5 . Therefore, there exists a constant $C_{1}$ such that $\int_{-\pi}^{\pi} P_{r}(\theta-r)\left|\phi(x) / \phi_{r}(x)\right|^{2} d x \leq C_{1}$, for $0 \leq r<1$ and $-\pi \leq \theta \leq \pi$.

(b) By an application of the Fejér-Riesz theorem, it can be assumed that the polynomial $Q$ is an analytic polynomial of degree $n$ with no zero inside the open unit disc. Then it is easy to show that with $Q_{r}=P_{r} * Q,\left|Q(\theta) / Q_{r}(\theta)\right| \leq C_{2}$, for some constant $C_{2}$ and all $0 \leq r<1$ and $-\pi \leq \theta \leq \pi$. Since $w=|Q|^{2} w_{0}=|Q \phi|^{2}$ and both $Q$ and $\phi$ are analytic functions, it follows that $P_{r} *(Q \phi)=Q_{r} \phi_{r}$ and $\int_{-\pi}^{\pi} P_{r}(\theta-x)\left|Q \phi(x) / Q_{r} \phi_{r}\right|^{2} d x \leq C_{1} C_{2}^{2}$, for all $0 \leq r<1$ and $-\pi \leq \theta \leq \pi$.

\section{REFERENCES}

1. J. L. Doob, Stochastic processes, Wiley, New York, 1953.

2. H. Helson and G. Szego, A problem in prediction theory, Ann. Mat. Pura Appl. (4) 51 (1960), $107-138$

3. H. Helson and D. Sarason, Past and future, Math. Scand. 21 (1967), 5-16.

4. A. N. Kolmogorov, Stationary sequences in a Hilbert space, Bull. Math. Univ. Moscow 2 (1941), $1-40$.

5. P. R. Masani, The prediction theory of multivariate stochastic processes. III, Acta Math. 104 (1960), 141-162.

6. M. Pourahmadi, A matricial extension of the Helson-Szego Theorem and its application in multivariate prediction, J. Multivariate Anal. (to appear).

7. M. Rosenblum, Summability of Fourier Series in $L^{p}(d \mu)$, Trans. Amer. Math. Soc. 105 (1962), $32-42$.

8. N. Wiener and P. Masani, The prediction theory of multivariate stochastic processes, Acta Math. 99 (1958), 93-137.

9. A. Zygmund, Trigonometric series. I, Cambridge Univ. Press, London and New York, 1959.

Department of MAthematical SCiEnCEs, NORThern Illinois University, DEKALB, ILLINOIS 60115

Current address: Center for Stochastic Processes, Department of Statistics, University of North Carolina, Chapel Hill, North Carolina 27514 